\title{
Interferon-Inducible Stat2 Activation of JUND and CLDN4: Mediators of IFN Responses
}

\author{
DANLIN JIA, RAMTIN RAHBAR, and ELEANOR N. FISH
}

\begin{abstract}
Signal transducer and activator of transcription 2 (Stat2) is a critical signaling protein involved in mediating interferon- $\alpha / \beta(I F N-\alpha / \beta)$ responses. Using site-directed mutagenesis in conjunction with gene microarray and biologic studies, we have previously demonstrated that in addition to Stat 2 functioning as a transactivator of transcription of a subset of IFN-inducible genes (ISG), Stat2-DNA binding mediates the transcriptional activation of other ISGs required for IFN-inducible antiviral and growth inhibitory responses. Among these, two candidate genes identified were Jun-D (JUND) and claudin-4 (CLDN4). To further explore the role of JUND and CLDN4 in IFN responses, we conducted knockdown studies using siRNA specific for either JUND or CLDN4 in 2fTGH fibroblast cells, and consistent with cells expressing the DNA-binding mutant of Stat2 (U6A2VV-II), siRNA-mediated knockdown resulted in cells that exhibited reduced antiproliferative and antiviral responses to IFN. Our data suggest that JUND and CLDN4 are critical mediators of the antiproliferative and antiviral effects of type I IFNs and further confirm the functional importance of the DNA-binding domain of Stat2.
\end{abstract}

I NTERFERON- $\alpha$ (IFN- $\alpha$ ) AND IFN- $\beta$ mediate diverse biologic effects, including the largely cell type-independent antiviral and antiproliferative responses and several cell type-restricted responses of immunologic relevance. ${ }^{1,2}$ High-affinity binding of an IFN to its cognate cell surface receptor, comprising of IFNAR-1 and IFNAR-2, leads to the activation of numerous intracellular signaling events. IFN-receptor interactions induce the phosphorylation-activation of the receptor-associated kinases Jak 1 and Tyk $2 .^{3}$ The Jaks then phosphorylate critical tyrosine residues in the intracellular regions of the receptor subunits that function as recruitment sites for specific transcription factors, Stats. The Stats are then phosphorylated by the Jaks, form homodimeric and heterodimeric complexes, and may also form complexes in association with other signaling effectors, including IFN regulatory factor-9 (IRF-9). ${ }^{4}$ Activated Stat complexes translocate into the nucleus, where they initiate transcription of IFN-inducible genes (ISGs) to modulate cellular functions. ${ }^{5}$

Stat2 activation plays a critical role in mediating IFN responses during infection, and not surprisingly, it is also one of the major targets of different viruses to evade an innate immune response. ${ }^{6,7}$ IFN-induced activation of Stat 2 results in the for- mation of distinct DNA-binding complexes: IFN-stimulated gene factor 3 (ISGF3) and Stat2:1/Stat2:3 heterodimer. ISGF3 is composed of Stat2:1 heterodimer in association with IRF-9. ISGF3-dependent complexes recognize the IFN-stimulated response element (ISRE) in the promoter of ISGs and mediate the transcription of effector proteins, including double-stranded RNA (dsRNA)-activated protein kinase R (PKR), 2' -5' -oligoadenylate synthetase ( $\left.2^{\prime} 5^{\prime} \mathrm{OAS}\right)$, and myxovirus resistance protein A (MxA). ${ }^{8}$ In contrast, ISGF3-independent Stat2:1 and Stat2:3 heterodimeric complexes bind to the $\gamma$-activated sequence (GAS)-like palindromic IFN-response element (pIRE) to mediate transcriptional activation of ISGs. ${ }^{9}$ Stat2-DNA binding activity is required for ISGF3-independent IFN-inducible responses. ${ }^{10}$ Cells expressing a mutant form of Stat2, which is unable to bind DNA once phosphorylated-activated by IFN, exhibit impaired antiviral and antiproliferative activities in response to IFN treatment. Comparison of the gene expression profiles from IFN-treated cells expressing either the intact or DNA-binding mutant of Stat2 revealed a subset of ISGs that were ISGF3 independent yet dependent on Stat2-DNA binding. ${ }^{11}$ Among the genes identified were Jun-D (JUND) and claudin-4 (CLDN4).

Division of Cellular and Molecular Biology, Toronto General Research Institute, University Health Network, and Department of Immunology, University of Toronto, Toronto, Canada. 
JUND is a member of the proto-oncogene JUN family, but unlike c-Jun whose expression is associated with progression through $\mathrm{S} / \mathrm{G}_{2}$ and $\mathrm{M}$ phases of the cell cycle, JUND has been shown to negatively regulate cell growth. ${ }^{12}$ Indeed, targeted gene deletion of JUND in mice revealed that JUND plays a significant role in suppressing cardiomyocyte hypertrophy. ${ }^{13}$

CLDN4, a member of the claudin family of proteins, is a 24$\mathrm{kDa}$ integral protein that has four transmembrane domains and is involved in homotypic interactions during the formation of tight junctions. ${ }^{14}$ The formation of tight junctions plays an important role in establishing the polarity of the endothelial lining, as well as regulating paracellular diffusion. CLDN4 has been implicated as a downstream target molecule in response to transforming growth factor- $\beta$ (TGF- $\beta$ ) and Ras/Raf/extracellular signal-regulated kinase pathways. ${ }^{15}$ Overexpression of CLDN4 has also been associated with reduction in the invasiveness and metastatic potential of pancreatic cancer cells.

Having determined that IFN-inducible Stat2-DNA binding contributes to the transcriptional activation of a subset of ISGs required for IFN-mediated antiviral and growth inhibitory responses, we provide evidence herein that CLDN4 and JUND, two such ISGs, participate in an IFN response.

$2 \mathrm{fTGH}$ cells expressing a DNA-binding mutant of Stat2 (U6A2VV-II) exhibit restricted IFN-inducible antiviral and growth inhibitory responses. ${ }^{10}$ Affymetrix gene microarray and subsequent gene validation studies revealed that U6A-2VV-II cells fail to upregulate gene expression for JUND and CLDN4 following IFN treatment. ${ }^{11}$ At the outset, we undertook studies to examine whether this reduction in gene expression is reflected in a reduction in corresponding protein expression. The data in Figure 1 show an IFNinducible upregulation of both JUND and CLDN4 in 2fTGH cells $16 \mathrm{~h}$ posttreatment, which is absent in U6A-2VV-II cells.

To investigate the contribution of JUND and CLDN4 to type I IFN-mediated growth inhibitory and antiviral responses, specific siRNA knockdown was used. Transfection of JUND-specific and CLDN4-specific siRNA duplexes, but not scrambled siRNA, in 2fTGH cells caused the reduction of JUND (Fig. 2A) and CLDN4 (Fig. 2B) expression $96 \mathrm{~h}$ posttransfection. Notably, when cells were transfected with both JUND-specific and CLDN4-specific siRNAs, the increased reduction in JUND and CLDN4 protein expression observed likely reflects the higher transfection efficiency associated with the longer incubation time with the siRNAs.

We next examined the contribution of JUND or CLDN4 to IFN- $\alpha / \beta$-mediated antiproliferative and antiviral activities. Parental 2fTGH, U6A-2VV-II, and 2fTGH cells transfected with either JUND-specific or CLDN4-specific or both siRNA were treated with IFN alfacon- 1 or IFN- $\beta$ for $96 \mathrm{~h}$. In contrast to parental $2 \mathrm{fTGH}$ cells, which were responsive to the antiproliferative effects of both IFN alfacon- 1 and IFN- $\beta$, U6A-2VV-

A
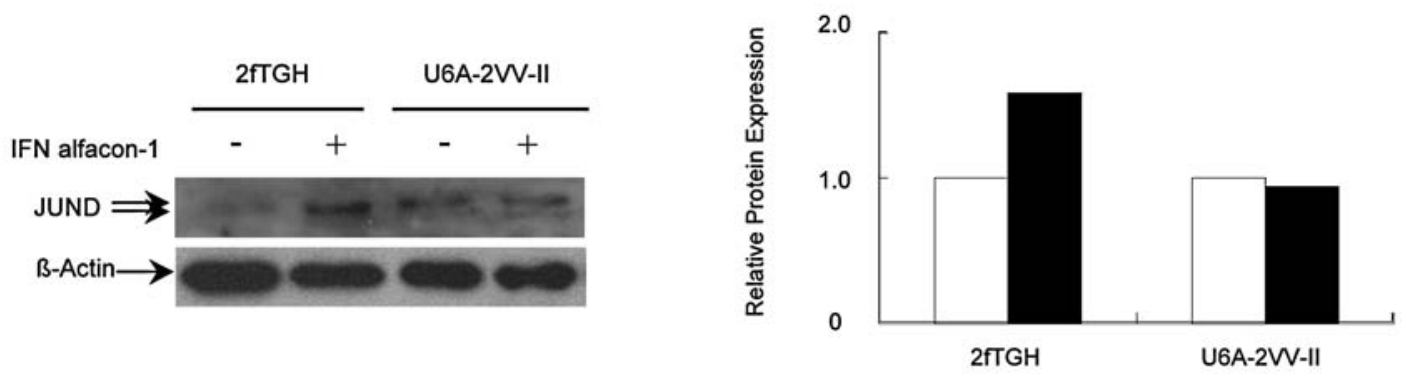

B
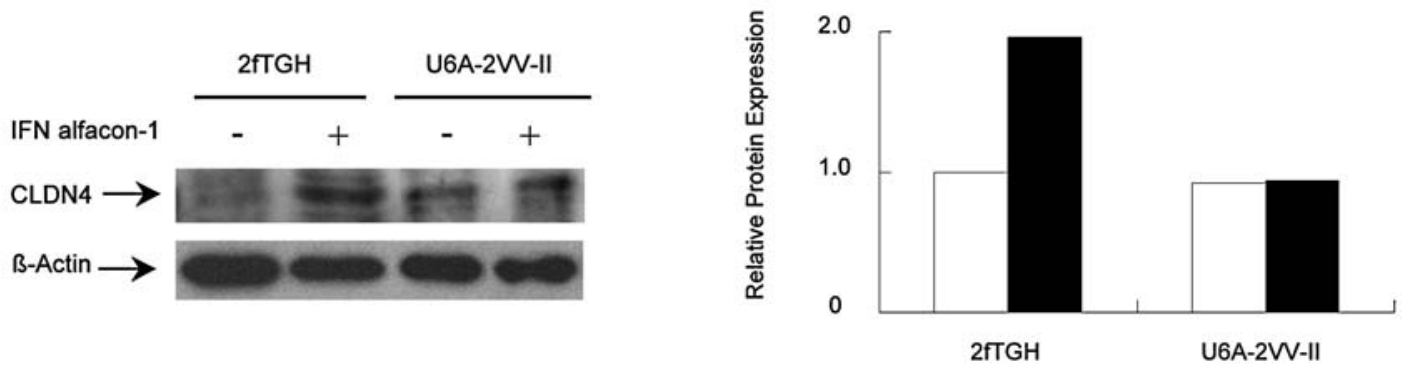

FIG. 1. IFN treatment of U6A-2VV-II cells fails to upregulate JUND and CLDN4. Human fibroblast 2fTGH cells were obtained from Dr. George Stark (Cleveland Clinic Foundation, Cleveland, OH). U6A-2VV-II cells expressing the Stat2-DNA binding mutant were generated as previously described. ${ }^{14} 2 \mathrm{fTGH}$ or U6A-2VV-II cells $\left(10^{6}\right)$ were either left untreated $(-)$ or treated with $10^{4} \mathrm{U} / \mathrm{mL}$ human recombinant IFN alfacon-1 (Intermune, Brisbane, CA) for $16 \mathrm{~h}(+)$ in Dulbecco's modified Eagle's medium (Invitrogen, Carlsbad, CA), supplemented with 10\% fetal bovine serum (FBS), $100 \mathrm{U} / \mathrm{mL}$ penicillin, $100 \mathrm{mg} / \mathrm{mL}$ streptomycin (Invitrogen). Cells were lysed and prepared for Western blot analysis as previously described. ${ }^{14}$ Membranes were immunoblotted with either (A) anti-JUND antibody (Santa Cruz, Santa Cruz, CA) to reveal the two isoforms of JUND (40 kDa and $35 \mathrm{kDa}$ ) or (B) anti-CLDN4 antibody (Santa Cruz). Membranes were stripped and reprobed with anti- $\beta$-actin antibody (Santa Cruz) to control for loading. The relative levels of protein expression are shown as fold induction over untreated controls in the right-hand panels: open bars, untreated; black bars, IFN treated. These data are representative of two independent experiments. 
A
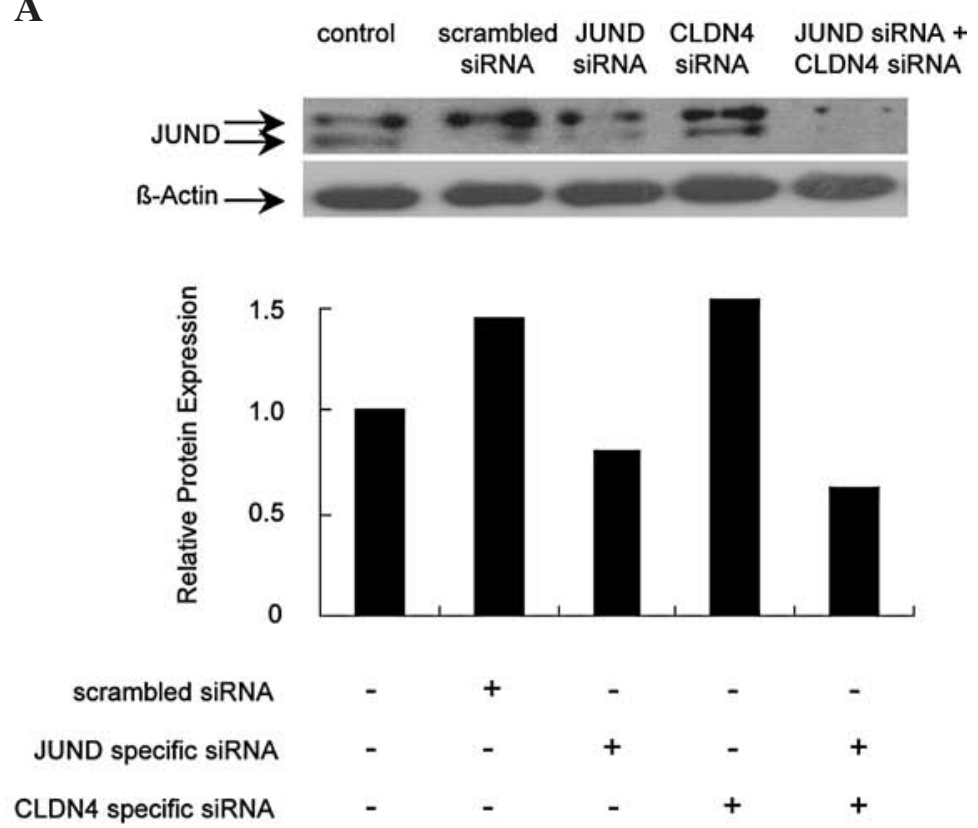

B
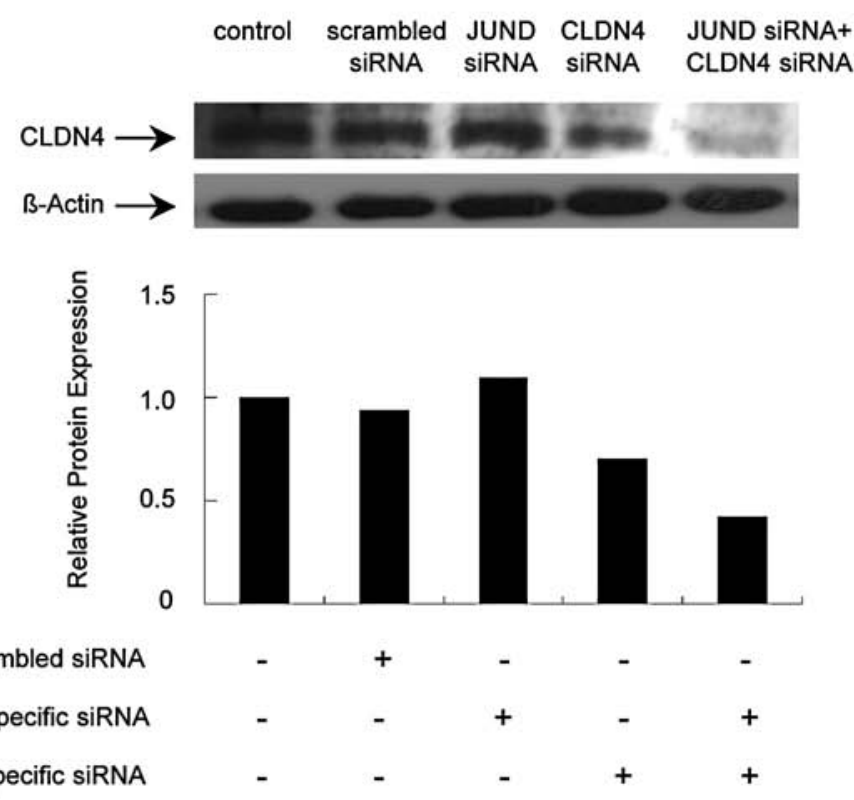

FIG. 2. Knockdown of JUND and CLDN4 in 2fTGH cells. JUND-specific and CLDN4-specific siRNA duplexes, as well as scrambled siRNA duplexes, were purchased from Qiagen (Chatsworth, CA). The JUND and CLDN4 target sequences were 5'AGGATGGAAACACCCTTCTA-3' and 5'-AGAGCCATATAACTGCTCAA-3', respectively. Prior to transfection, $8 \times 10^{5}$ $2 \mathrm{fTGH}$ cells were seeded in 60 -mm cell culture plates in $4 \mathrm{~mL} 2 \%$ FBS-containing DMEM. The appropriate siRNA (20 nM) (4 $\mu \mathrm{L}$ ) was incubated with HiPerfect transfection buffer (Qiagen) according to the manufacturer's specifications. 2fTGH cells were transfected with either scrambled siRNA or with siRNA specific for JUND or CLDN4 or both. 2fTGH cells treated with transfection buffer alone were included as controls. Cell lysates were prepared $96 \mathrm{~h}$ posttransfection, and proteins were resolved by SDS-PAGE and immunblotted for (A) JUND or (B) CLDN4, using antibodies described in Figure 1. The levels of protein expression indicated are relative to untreated controls.

II cells were shown to be less responsive, as anticipated. More importantly, 2fTGH cells with decreased expression of JUND or CLDN4 or both exhibited reduced sensitivity to the antiproliferative effects of IFN alfacon-1 and IFN- $\beta$ (Fig. 3).
When parental 2fTGH cells U6A-2VV-II cells, and the $2 \mathrm{fTGH}$ cells expressing reduced levels of CLDN4 or JUND were assessed for their responsiveness to IFN-inducible antiencephalomyocarditis virus (EMCV) activity, similar results 
A

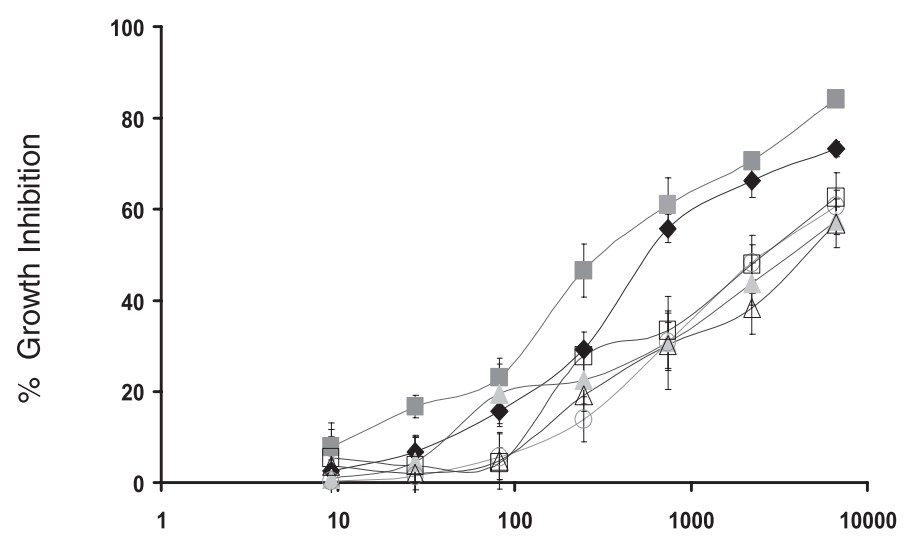

IFN-alfacon-1 (U/mL)

B

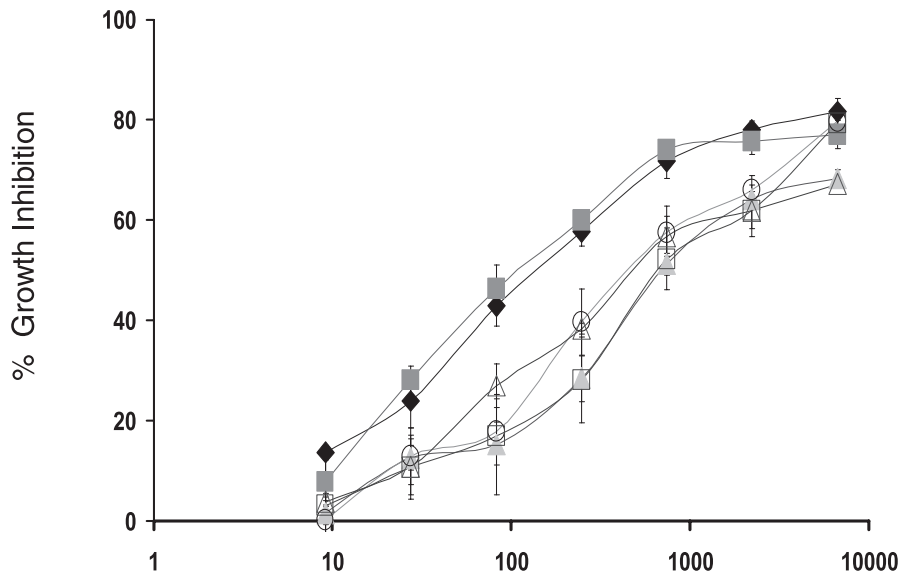

IFN- $\beta(\mathrm{U} / \mathrm{mL})$

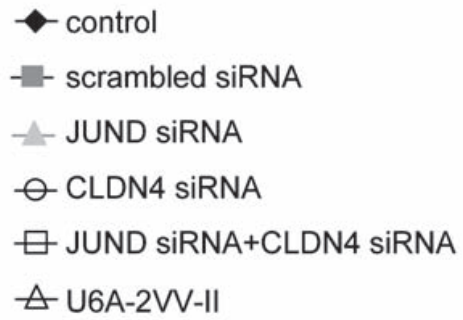

FIG. 3. JUND and CLDN4 are important for IFN alfacon-1 and IFN- $\beta$-mediated growth inhibitory responses. 2fTGH, U6A2VV-II, or 2fTGH cells transfected with either scrambled siRNA or JUND-specific or CLDN4-specific siRNA, or both, were either left untreated or treated with serial dilutions of (A) IFN alfacon-1 or (B) IFN- $\beta$ (provided by Darren Baker, Biogen Inc., MA) for $96 \mathrm{~h}$. Cell proliferation was quantitated using a crystal violet colorimetric assay, as previously described. ${ }^{14}$ Data are expressed as percent inhibition compared with untreated cells. Mean value \pm SE of three independent experiments is shown.

were obtained. Specifically, both IFN alfacon- 1 and IFN- $\beta$ induced strong protective effects in parental $2 \mathrm{fTGH}$ but not in U6A-2VV-II cells against EMCV. 2fTGH cells transfected with either JUND-specific or CLDN4-specific siRNA or both were less responsive to the antiviral effects of the IFNs (Fig. 4). Notably, we observe that simultaneous knockdown of JUND and CLDN4 expression resulted in an additive effect in terms of restricted responsiveness to IFN-mediated antiviral activity.

In summary, we have extended our earlier studies to specifically interrogate the effects of two IFN-inducible ISGs whose expression is regulated by Stat2-DNA binding. Focusing on CLDN4 and JUND, we provide evidence for their contribution to IFN- $\alpha / \beta$-inducible growth inhibitory and anti-EMCV effects. 
A

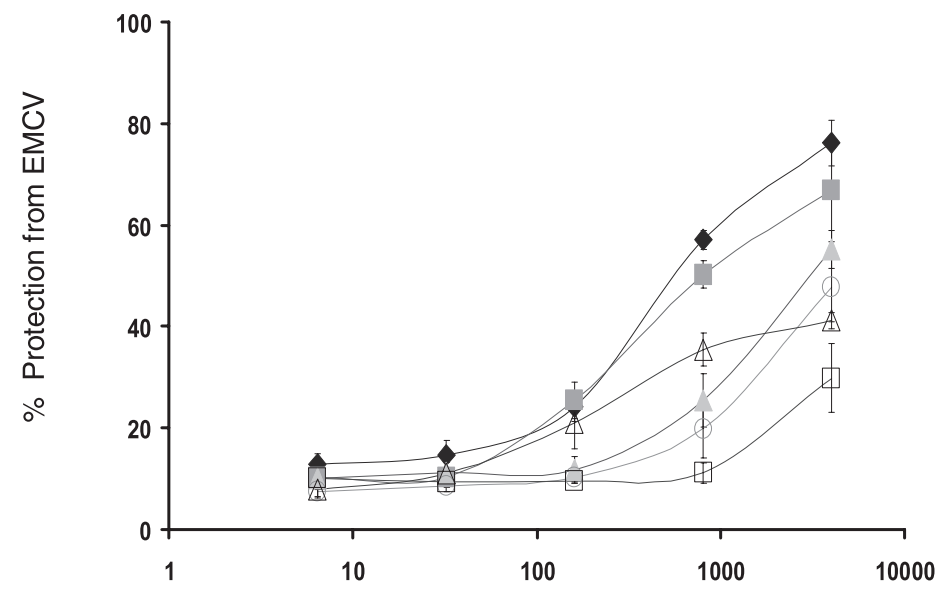

IFN-alfacon-1 (U/mL)

\section{B}

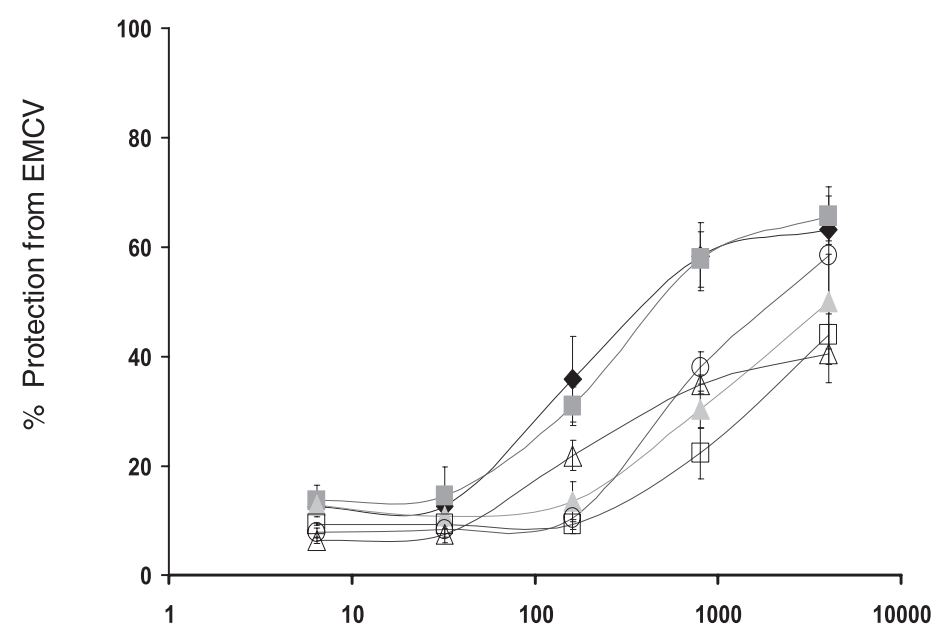

IFN- $\beta(\mathrm{U} / \mathrm{mL})$

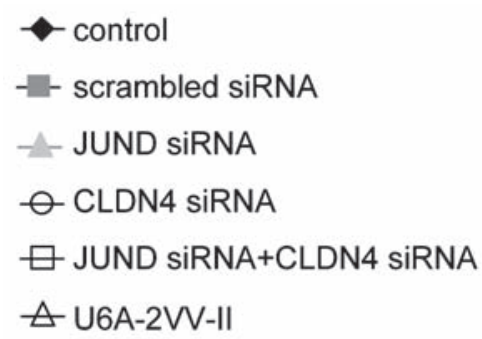

FIG. 4. JUND and CLDN4 are important for IFN alfacon- 1 and IFN- $\beta$-mediated anti-EMCV responses. 2fTGH, U6A-2VVII, or $2 \mathrm{fTGH}$ cells transfected with either scrambled siRNA or JUND-specific or CLDN4-specific siRNA or both were treated with serial dilutions of (A) IFN alfacon-1 or (B) IFN- $\beta$ for $16 \mathrm{~h}$ and then challenged with EMCV. Twenty-four hours later, virusinduced cytopathic effects (CPE) were quantitated using a crystal violet colorimetric assay as previously described. ${ }^{14}$ Data are expressed as percent protection from the CPE of EMCV compared with untreated, uninfected cells. Mean value \pm SE of three independent experiments is shown.

Overexpression of JUND leads to a reduction in cell growth and accumulation of cells in $\mathrm{G}_{\mathrm{o}} / \mathrm{G}_{1} \cdot{ }^{12} \mathrm{It}$ has been suggested that JUND may exert its growth inhibitory functions through its association with p202. Activation of p202 can inhibit the tran- scriptional activation of genes required for $\mathrm{S}$ phase and negatively regulate cell growth. ${ }^{16,17}$ IFNs- $\alpha / \beta$ may, therefore, exert their growth inhibitory effects in cells in part through the upregulation of JUND expression. In addition to influencing cell 
proliferation, increases in JUND expression can lead to elevation of apoptotic factors, such as Bax and caspase-3, during cellular stress. ${ }^{18,19}$ The protective effect of JUND during EMCV infection may be derived from apoptosis induction, thereby preventing viral replication.

Similar to JUND, our data suggest the importance of IFNinducible expression of CLDN4 in IFN- $\alpha / \beta$-mediated growth inhibitory responses. Studies in F9 murine embryonic carcinoma cells line have shown that antiproliferative effects induced by doxycycline-inducible hepatocyte nuclear factor- $4 \alpha$ (HNF$4 \alpha$ ) are accompanied by upregulation of tight junction proteins occludin, CLDN6, and CLDN7, as well as the formation of functional tight junctions. ${ }^{20}$ Similarly, overexpression of another cell-cell adhesive protein, H-cadherin, can also inhibit proliferation in a junction-dependent manner. In other mouse studies, overexpression of $\mathrm{H}$-cadherin was shown to inhibit both tumor growth and migration by inducing expression of $\mathrm{p} 21 .^{21,22}$ Viewed altogether, we infer that CLDN4 may inhibit cell proliferation by similar mechanisms.

Tight junctions are critical for limiting tissue permeability. Components of tight junction are frequently targeted by infectious pathogens as a means to enter host tissues. ${ }^{23-26}$ Adenoviruses disrupt cellular tight junctions to initiate viral release. ${ }^{27}$ IFN-induced CLDN4 may exert its antiviral effect in part by reinforcing the integrity of tight junctions, as a means to prevent dissemination of virions into surrounding tissues. Recently, Fedwick et al. ${ }^{28}$ showed that Helicobacter pylori-induced phosphorylation of myosin light chains can disrupt both CLDN4 and CLDN5 expression, thereby increasing the permeability of epithelial cells, which is associated with increased severity of $H$. pylori infection. Alteration of other tight junction factors, such as ZO, occludin, CLDN1, and CLDN5, also leads to changes in the permeability of the endothelial lining, implicated in the pathogenesis of HIV-1 encephalitis (HIVE) and HIV-associated dementia (HAD). ${ }^{29,30}$

The preceding provides evidence for the specific regulation by IFN of two ISGs whose transcriptional activation is determined by Stat2-DNA binding. These data provide further confirmation for Stat2-mediated, ISGF3-independent regulation of gene expression that is critical for IFN- $\alpha / \beta$-induced biologic responses. Moreover, our findings shed light on two critical effectors of IFN-induced responses, namely, CLDN4 and JUND.

\section{ACKNOWLEDGMENT}

These studies were supported by a Canadian Institutes of Health Research Grant MOP 15094 to E.N.F.

\section{REFERENCES}

1. Stark GR, Kerr IM, Williams BR, Silverman RH, Schreiber RD. How cells respond to interferons. Annu. Rev. Biochem. 1998;67: 227-264.

2. Pestka S, Krause CD, Walter MR. Interferons, interferon-like cytokines, and their receptors. Immunol. Rev. 2004;202:8-32.

3. Brierley MM, Fish EN. IFN- $\alpha / \beta$ receptor interactions to biologic outcomes: understanding the circuitry [Review]. J. Interferon Cytokine Res. 2002;22:835-845.
4. Fu XY, Kessler DS, Veals SA, Levy DE, Darnell JE Jr. ISGF3, the transcriptional activator induced by interferon alpha, consists of multiple interacting polypeptide chains. Proc. Natl. Acad. Sci. USA 1990;87:8555-8559.

5. Platanias LC. Mechanisms of type-I and type II-interferon-mediated signalling [Review]. Nat. Rev. Immunol. 2005;5:375-386.

6. Jones M, Davidson A, Hibbert L, Gruenwald P, Schlaak J, Ball S, Foster GR, Jacobs M. Dengue virus inhibits alpha interferon signaling by reducing Stat2 expression. J. Virol. 2005;79:5414-5420.

7. Hahm B, Trifilo MJ, Zuniga EI, Oldstone MB. Viruses evade the immune system through type I interferon-mediated Stat2-dependent, but Stat1-independent, signaling. Immunity 2005;22:247-257.

8. Samuel CE. Antiviral actions of interferons [Review]. Clin. Microbiol. Rev. 2001;14:778-809.

9. Ghislain JJ, Fish EN. Application of genomic DNA affinity chromatography identifies multiple interferon-alpha-regulated Stat2 complexes. J. Biol. Chem. 1996;271:12408-12413.

10. Brierley MM, Fish EN. Functional relevance of the conserved DNA-binding domain of Stat2. J. Biol. Chem. 2005;280: 13029-13036.

11. Brierley MM, Marchington KL, Jurisica I, Fish EN. Identification of GAS-dependent interferon-sensitive target genes whose transcription is Stat2-dependent but ISGF3-independent. FEBS $J$. 2006;273:1569-1581.

12. Pfarr CM, Mechta F, Spyrou G, Lallemand D, Carillo S, Yaniv M. Mouse JunD negatively regulates fibroblast growth and antagonizes transformation by ras. Cell 1994;76:747-760.

13. Hilfiker-Kleiner D, Hilfiker A, Kaminski K, Schaefer A, Park JK, Michel K, Quint A, Yaniv M, Weitzman JB, Drexler H. Lack of JunD promotes pressure overload-induced apoptosis, hypertrophic growth, and angiogenesis in the heart. Circulation 2005;112: 1470-1477.

14. Tsukita S, Furuse M. Claudin-based barrier in simple and stratified cellular sheets [Review]. Curr. Opin. Cell. Biol. 2002;14:531-536.

15. Michl P, Barth C, Buchholz M, Lerch MM, Rolke M, Holzmann KH, Menke A, Fernsterer H, Giehl K, Lohr M, Leder G, Iwamura T, Adler G, Gress TM. Claudin-4 expression decreases invasiveness and metastatic potential of pancreatic cancer. Cancer Res. 2003;63:6265-6271.

16. Xin H, Pramanik R, Choubey D. Retinoblastoma ( $R b)$ protein upregulates expression of the Ifi202 gene encoding an interferon-inducible negative regulator of cell growth. Oncogene 2003;22: 4775-4785.

17. Gutterman JU, Choubey D. Retardation of cell proliferation after expression of p202 accompanies an increase in p21(WAF1/CIP1). Cell Growth Differ. 1999;10:93-100.

18. Tsuji Y. JunD activates transcription of the human ferritin $\mathrm{H}$ gene through an antioxidant response element during oxidative stress. Oncogene 2005;24:7567-7578.

19. Li T, Lu L. Ultraviolet-induced JunD activation and apoptosis in myeloblastic leukemia ML-1 cells. J. Biol. Chem. 2002;277: 32668-32676.

20. Chiba H, Itoh T, Satihisa S, Sakai N, Noguchi H, Osanai M, Kojima T, Sawada N. Activation of p21CIP1/WAF1 gene expression and inhibition of cell proliferation by overexpression of hepatocyte nuclear factor-4alpha. Exp. Cell. Res. 2005;302:11-21.

21. Zhong Y, Lopez-Barcons L, Haigentz M Jr, Ling YH, Perez-Soler R. Exogenous expression of $\mathrm{H}$-cadherin in $\mathrm{CHO}$ cells regulates contact inhibition of cell growth by inducing p21 expression. Int. J. Oncol. 2004;24:1573-1579.

22. Lee SW, Reimer CL, Campbell DB, Cheresh P, Duda RB, Kocher $\mathrm{O}$. H-Cadherin expression inhibits in vitro invasiveness and tumor formation in vivo. Carcinogenesis 1998;19:1157-1159.

23. Zheng J, Xie Y, Campbell R, Song J, Massachi S, Razi M, Chiu $\mathrm{R}$, Berenson J, Yang OO, Chen IS, Pang S. Involvement of claudin7 in HIV infection of CD4- cells. Retrovirology 2005;2:79. 
24. Bergelson JM, Cunningham JA, Droguett G, Kurt-Jones EA, Krithivas A, Hong JS, Horwitz MS, Crowell RL, Finberg RW. Isolation of a common receptor for Coxsackie B viruses and adenoviruses 2 and 5. Science 1997;275:1320-1323.

25. Barton ES, Forrest JC, Connolly JL, Chappell JD, Liu Y, Schnell FJ, Nusrat A, Parkos CA, Dermody TS. Junction adhesion molecule is a receptor for reovirus. Cell 2001;104:441-451.

26. Geraghty RJ, Krummenacher C, Cohen GH, Eisenberg RJ, Spear PG. Entry of alphaherpesviruses mediated by poliovirus receptorrelated protein 1 and poliovirus receptor. Science 1998;280: $1618-1620$.

27. Walters RW, Freimuth P, Moninger TO, Ganske I, Zabner J, Welsh MJ. Adenovirus fiber disrupts CAR-mediated intercellular adhesion allowing virus escape. Cell 2002;110:789-799.

28. Fedwick JP, Lapointe TK, Meddings JB, Sherman PM, Buret AG. Helicobacter pylori activates myosin light-chain kinase to disrupt claudin-4 and claudin-5 and increase epithelial permeability. Infect. Immun. 2005;73:7844-7852.

29. Persidsky Y, Heilman D, Haroah J, Zelivyanskaya M, Persidsky R, Weber GA, Shimokawa H, Kaibuchi K, Ikezu T. Rho-mediated regulation of tight junctions during monocyte migration across the blood-brain barrier in HIV-1 encephalitis (HIVE). Blood 2006; 107:4770-4780.

30. Kanmogne GD, Primeaux C, Grammas P. HIV-1 gp120 proteins alter tight junction protein expression and brain endothelial cell permeability: implications for the pathogenesis of HIV-associated dementia. J. Neuropathol. Exp. Neurol. 2005;64:498-505.

Address reprint requests or correspondence to:

Dr. Eleanor N. Fish

Division of Cellular and Molecular Biology

Toronto General Research Institute

67 College Street

Toronto, ON M5G $2 \mathrm{MI}$

Canada

Tel: 416-340-5380

Fax: 416-340-3453

E-mail: en.fish@utoronto.ca

Received 24 January 2006/Accepted 6 February 2007 
